\title{
The Complex Optical to Soft X-Ray Spectrum of Low-Redshift Radio-quiet Quasars. II. Comparison with Free-Free and Accretion Disk Models
}

\section{Citation}

Fiore, Fabrizio, Martin Elvis, Aneta Siemiginowska, Belinda J. Wilkes, Jonathan C. McDowell, and Smita Mathur. 1995. "The Complex Optical to Soft X-Ray Spectrum of Low-Redshift Radio-Quiet Quasars. II. Comparison with Free-Free and Accretion Disk Models." The Astrophysical Journal 449 (August): 74 . doi:10.1086/176033.

\section{Published Version}

doi:10.1086/176033

\section{Permanent link}

http://nrs.harvard.edu/urn-3:HUL.InstRepos:30212149

\section{Terms of Use}

This article was downloaded from Harvard University's DASH repository, and is made available under the terms and conditions applicable to Other Posted Material, as set forth at http:// nrs.harvard.edu/urn-3:HUL.InstRepos:dash.current.terms-of-use\#LAA

\section{Share Your Story}

The Harvard community has made this article openly available.

Please share how this access benefits you. Submit a story.

Accessibility 


\title{
THE COMPLEX OPTICAL TO SOFT X-RAY SPECTRUM OF LOW-REDSHIFT RADIO-QUIET QUASARS. ${ }^{1}$ II. COMPARISON WITH FREE-FREE AND ACCRETION DISK MODELS
}

\author{
Fabrizio Fiore, ${ }^{2}$ Martin Elvis, Aneta Siemiginowska, Belinda J. Wilkes, \\ Jonathan C. McDowell, and Smita Mathur \\ Harvard-Smithsonian Center for Astrophysics, 60 Garden Street, Cambridge, MA 02138 \\ Received 1994 March 31; accepted 1995 February 17
}

\begin{abstract}
We compare the optical to soft X-ray spectral energy distributions (SEDs) of a sample of bright low-redshift $(0.048<z<0.155)$, radio-quiet quasars, with a range of thermal models which have been proposed to explain the optical/UV/soft X-ray quasar emission: (1) optically thin emission from an ionized plasma, (2) optically thick emission from the innermost regions of an accretion disk in Schwarzschild and Kerr geometries. We presented ROSAT PSPC observations of these quasars in an earlier paper. Here our goals are to search for the signature of thermal emission in the quasar SEDs, and to investigate whether a single component is dominating at different frequencies.

We find that isothermal optically thin plasma models can explain the observed soft X-ray color and the mean optical-ultraviolet (OUV) color. However, they predict an ultraviolet (1325 $\AA$ ) luminosity a factor of 3 to 10 times lower than observed. Pure disk models, even in a Kerr geometry, do not have the necessary flexibility to account for the observed OUV and soft X-ray luminosities. Additional components are needed both in the optical and in the soft X-rays (e.g., a hot corona can explain the soft X-ray color). The most constrained modification of pure disk models, is the assumption of an underlying power-law component extending from the infrared $(3 \mu \mathrm{m})$ to the X-ray. This can explain both the OUV and soft X-ray colors and luminosities and does not exceed the $3 \mu \mathrm{m}$ luminosity, where a contribution from hot dust is likely to be important. We also discuss the possibility that the observed soft X-ray color and luminosity are dominated by reflection from the ionized surface of the accretion disk.

While modifications of both optically thin plasma models and pure disk models might account for the observed SED, we do not find any strong evidence that the OUV bump and soft X-ray emission are one and the same component. Likewise, we do not find any strong argument which definitely argues in favor of thermal models.
\end{abstract}

Subject headings: quasars: general - X-rays: galaxies

\section{INTRODUCTION}

The most striking property of the continuum of unbeamed quasars (simply quasars hereafter) is the fact that it extends over at least seven decades in frequency, from $100 \mu \mathrm{m}$ to 100 $\mathrm{keV}$, maintaining a similar luminosity per decade. The most prominent feature of a quasar's spectral energy distribution (SED) is the blue bump which dominates the optical/ ultraviolet (OUV) emission and contains $\gtrsim 50 \%$ of the energy output. The blue bump could have a broad maximum in the 1300-1000 $\AA$ range, as suggested by Malkan (1988), or could extend into the so far unobserved extreme-UV region, if some intrinsic reddening is present. The observed soft X-ray flux is in most cases lower than that predicted by extrapolating the UV flux with the typical spectral indices measured at wavelengths longer than $\sim 1000 \AA$. This requires a sharp drop in the extreme UV region. The soft X-ray flux ( $\lesssim 1 \mathrm{keV})$, however, is higher than the extrapolation of the higher energy $(2-10 \mathrm{keV})$ power law. This low-energy $\mathrm{X}$-ray turn-up, commonly known as the soft $\mathrm{X}$-ray excess, is generally interpreted as the high-

\footnotetext{
1 This paper is based in part on data obtained on the Multiple Mirror Telescope (MMT), a joint facility of the Smithsonian Institution and the - University of Arizona.

2 Postal address: Osservatorio Astronomico di Roma, via dell'Osservatorio 5, Monteporzio-Catone (RM), I00040 Italy; fiore@cfa.harvard.edu.
}

energy tail of the blue bump. Although such a connection is suggestive given that both components increase into the unobservable extreme-UV, no direct evidence yet exists. However it is clear that the combination of OUV and soft X-ray data will provide invaluable constraints on models which seek to explain both components, such as thermal emission from an accretion disk (e.g., Shields 1978; Malkan \& Sargent 1982; Czerny \& Elvis 1987; Sun \& Malkan 1989; and Laor 1990, on the OUV side; Turner \& Pounds 1988 and Elvis et al. 1991, from the X-ray side), and optically thin free-free (see Barvainis 1993 for a review).

The soft $\mathrm{X}$-ray excess was recognized as a distinct and common component below $1 \mathrm{keV}$ of the X-ray spectra of Seyfert 1 galaxies and quasars in both EXOSAT and Einstein data (Turner \& Pounds 1989; Wilkes \& Elvis 1987; Masnou et al. 1992). However, the poor energy resolution of both instruments at these energies resulted in little or no information about the spectral form. The softer energy band and improved resolution of the ROSAT (Trümper 1983) PSPC (Pfeffermann et al. 1987) provides us with our first opportunity to study the spectrum of the soft excess and thus to realize its full potential as a constraint on models for the OUV/X-ray continuum.

We observed a sample of low-redshift, radio-quiet quasars selected to be the brightest with known soft excesses, to acquire high signal-to-noise $(>100 \sigma)$ spectra with the PSPC. The analysis of these X-ray observations is reported in a compan- 
ion paper (Fiore et al. 1994, hereafter Paper I). The main results from this paper are the following:

1. The PSPC spectra span a broad range of slopes $(1.3<$ $\alpha_{E}<2.3$, where $\left.F_{v} \propto v^{-\alpha_{E}}\right)$ and are steeper than at higher energies (typically $2-10 \mathrm{keV}$ ) by $\Delta \alpha_{E}=0.5-1$. They are dominated by the soft X-ray excess.

2. The deviations from a single power-law model in the $0.2-2 \mathrm{keV}$ band are small but still significant. The "break point" between the hard and soft components is in all cases above $0.7 \mathrm{keV}$. The PSPC spectra exclude "narrow" models, such as a single line or a blackbody, for the soft X-ray component.

3. The strength of any line emission feature in the soft X-ray band is small (less than $10 \%-20 \%$ of the counts in each channel), unless the continuum is complicated.

Here we present the optical to soft X-ray SEDs of the quasars and compare them with the predictions of a wide range of thermal models which have been proposed to explain the OUV/soft X-ray quasar emission: (1) optically thin plasma emission, (2) optically thick thermal emission from the innermost regions of an accretion disk in Schwarzschild and Kerr geometries. Our aims are to search for the signature of thermal emission in the quasar SEDs and to investigate whether a single component is dominating at different frequencies or rather if the quasar SEDs are best reproduced by the superposition of different components.

\section{THE SOFT X-RAY COMPONENT AND THE BIG BLUE BUMP}

A longstanding question in quasar research is whether the soft X-ray emission and the OUV blue bump are one and the same component. The most direct way to verify this is to search for simultaneous/correlated variations in both the UV and soft X-ray emission. Unfortunately simultaneous UV and soft X-ray observations of longer than a few hours are difficult to schedule with ROSAT and IUE and have so far been performed only for a few, very bright objects like the BL Lacertae object PKS 2155-304 (Edelson et al. 1995).

A number of quasars have been observed nearly simultaneously by IUE and ROSAT during the ROSAT All-Sky Survey (Walter et al. 1995). These authors found that in six out of eight cases the shape of the UV to soft X-ray component is similar, while the relative strength of the UV emission compared to the hard X-ray power law varies by a factor of 10 over the sample.

A more indirect method is through the statistical properties of the UV and soft X-ray emission of a sample of quasars. Walter \& Fink (1993) have reported a strong correlation between the soft X-ray (PSPC) spectral indices and the ratio between the flux at $1350 \AA$ and that at $2 \mathrm{keV}$ in a sample of about 50 quasars. Under the assumption that the PSPC spectral index is a good indicator of the strength of the soft excess, this was interpreted as evidence that the soft X-ray emission and OUV emission are part of the same component: a O-UV$\mathrm{X}$-ray bump. There are some concerns about this analysis. We note that:(1) the Walter and Fink sample is soft X-ray selected, since it includes only the brightest quasars in the ROSAT All-Sky Survey. Then, the PSPC spectral index and the $2 \mathrm{keV}$ flux are strongly correlated quantities, since $2 \mathrm{keV}$ is the upper end of the PSPC band: in a flux-limited soft X-ray survey a source with a low $2 \mathrm{keV}$ flux is likely to have also a steep PSPC spectral index. (2) The Walter and Fink sample is very heterogeneous. It contains: low-luminosity Seyfert galaxies and high-luminosity, high-redshift quasars, sources with strong and faint blue bumps; radio-loud and radio-quiet objects; strongly absorbed and unabsorbed sources. The correlations between these properties and the luminosity in the optical, UV, and soft . $\mathrm{X}$-ray bands are all complicated and could drive, at least in part, the Walter and Fink correlation.

Laor et al. (1994) report a result similar to the Walter and Fink correlation. They found a significant correlation between the PSPC spectral index and the optical (3000 $\AA)$ to X-ray $(2 \mathrm{keV})$ rest frame spectral index $\alpha_{\text {ox }}$ in a sample of 10 optically selected, $z<0.4(\langle z\rangle=0.22), M_{B}<-23$ quasars (three of which are radio-loud). Being optically selected, this small sample does not suffer from the selection bias mentioned above.

As an example we plot in Figure $1 \alpha_{\mathrm{OX}}$ as a function of the PSPC best-fit single power-law energy index $\alpha_{E}{ }^{3}$ We include the Laor et al. (1994) radio quiet objects, the sample presented in Paper I (four objects are PG quasars, and the remaining two, NAB $0205+024$ and Mrk 205 have a comparably strong blue bump), and all the radio-quiet PG quasars in the Walter and Fink sample which have small Galactic $N_{\mathrm{H}}$ and $z<0.4$. The combined sample consists of 22 objects, all radio-quiet, with a strong blue bump, without significant intrinsic absorption and spanning a large range in optical luminosity $\left(-26<M_{B}<\right.$ -21 ). We estimate that a spread in $\alpha_{O X}$ of \pm 0.1 is introduced by the fact that the observations in OUV and X-ray are not simultaneous (from the data in Tables 1 and 2, see below). It is not clear whether or not the amplitude of the long-term UV variability is correlated with the luminosity. O'Brien et al. (1988) found some evidence for an inverse correlation, but their sample of high luminosity objects was small. For the sample presented in this paper there is no evidence for a correlation between the amplitude of the UV variability and luminosity. We therefore assume that the spread in $\alpha_{O X}$ is roughly similar at all luminosities. For the objects in the sample presented in this paper the optical luminosity has been corrected for the

${ }^{3} \alpha_{\mathrm{ox}}=\frac{\log [L(2500 \AA) / L(2 \mathrm{keV})]}{2.6057}$

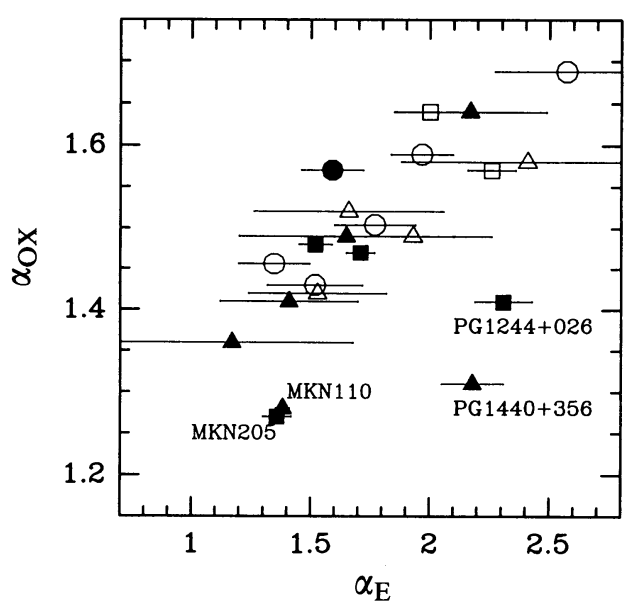

FIG. 1.-PSPC energy index plotted against the OUV to X-ray energy index $\alpha_{\mathrm{OX}}$ for the quasars of the sample presented in this paper (squares), the radio-quiet quasars in the Laor et al. (1994) sample (circles), and the PG objects in the sample of Walter \& Fink (1993) with $z<0.4$ (triangles). Open symbols identify high-luminosity quasars $\left(M_{B}<-23.5\right)$, filled symbols identify low-luminosity quasars $\left(M_{B}>-23.5\right)$. 
contribution from the host galaxy, while the objects in the Laor et al. and Walter and Fink samples were not so corrected. We note however that the contribution of the host galaxy should be small even in low-luminosity objects at $3000 \AA$ (see the galaxy template in Elvis et al. 1994a), thus affecting $\alpha_{\text {ox }}$ only slightly.

The correlation between $\alpha_{E}$ and $\alpha_{O X}$ is quite good for the high optical luminosity objects (11 objects with $M_{B}<-23.5$, linear correlation coefficient equal to 0.855 corresponding to a probability of $99.95 \%$ ). The linear correlation coefficient for the whole sample is lower $(r=0.60$, corresponding to a probability of $99.6 \%$ ). In fact, the four points which deviate most from the correlation are all lower optical luminosity sources. Two of these sources, PG $1440+356$ (Mrk 478) and PG $1244+026$ have a very steep PSPC slope but their soft X-ray luminosity is comparable or even higher than their UV luminosity, unlike all the other sources in the sample. PG $1440+356$ is one of the few quasars detected at $\sim 0.1 \mathrm{keV}$ by the ROSAT Wide Field Camera (Pounds et al. 1993). Another Wide Field Camera source (RE 1034+398) has a very steep PSPC spectrum (Puchnarewicz et al. 1995), flat $\alpha_{\text {Ox }}$ and soft X-ray luminosity higher than the UV luminosity. Pounds (1994) and Fiore \& Eivis (1995) suggested that these sources could belong to a separate class of quasars, sources that emit the most of the power in soft X-rays, unlike the majority of optically selected quasars. Fiore \& Elvis (1995) suggested that these source could resemble Galactic black hole candidates in the high (and soft) state.

Assuming that the PSPC spectrum of radio-quiet, strong blue bump sources, is made up of two distinct components, a steep one dominating below $\sim 1-2 \mathrm{keV}$ and a hard one dominating at higher energies, the correlation in Figure 1 can be interpreted in two ways. Either (1) the intensity of the soft component is more or less the same in all sources while that of the hard one varies over a broad range, or (2) the intensity of the soft component varies over a broad range in these sources while that of the hard one is more or less the same. The significantly larger scatter in the $2 \mathrm{keV}$ luminosity found by Laor et al. (1994) with respect to that in the $0.3 \mathrm{keV}$ luminosity, argues for the first case. This suggests that, in high optical luminosity sources at least, a correlation between the blue bump and the soft X-ray component, does indeed exist. However, this does not prove that the luminosity in different frequency bands arises from the same emission component. In the following sections we test the single component hypothesis by discussing the ability of various models to reproduce both X-ray and OUV data.

\section{OBSERVATIONAL DATA}

\subsection{X-Ray Data}

The numerical results of the spectral fitting from Paper I are given in Table 1 . Table 1 lists the soft $\mathrm{X}$-ray monochromatic luminosities $v L_{v}$ at $0.4 \mathrm{keV}$ and $1 \mathrm{keV}$ (soft component only), the soft component energy index $\left(F_{v} \propto v^{-\alpha_{E S}}\right)$, and $2 \mathrm{keV}$ monochromatic luminosity (soft plus hard components) for the quasars in our sample. They are from Paper I and were obtained by fitting to the PSPC data a two power-law model, or a power law plus thermal bremsstrahlung model, with low energy absorption $\left(N_{\mathrm{H}}\right.$ was limited to values greater than the Galactic column along the line of sight). The power law plus thermal bremsstrahlung model gives a fit of quality comparable to the two power-law model in all sources but PG $1426+015$, where it produces a significantly worse $\chi^{2}$ (see Paper I). Errors on the luminosities represent $1 \sigma$ confidence intervals for four interesting parameters.

In Paper I we compared the PSPC results concerning the soft component intensity with those obtained with previous satellites, finding that they always agree within the errors: In spite of the low energy resolution of the EXOSAT LE and the Einstein IPC several authors estimated the shape of the soft $\mathrm{X}$-ray component in a number of quasars. In particular, Comastri et al. (1992) fitted the EXOSAT ME/LE data with a two power-law model (with $N_{\mathrm{H}}$ fixed to the Galactic value) for a sample of six quasars, three of which are in common with our sample. They found a soft component energy index $\alpha_{E S}$ of $3.33_{-0.42}^{+0.66}$ in PG $1211+143,3.32_{-0.67}^{+1.20}$ in TON 1542 (PG $1229+204)$, and $2.66_{-0.49}^{+1.09}$ in PG $1426+015$. For each source the EXOSAT indices and PSPC indices in Table 1 are consis-

TABLE 1

X-Ray Observations

\begin{tabular}{|c|c|c|c|c|c|}
\hline Name & $v L_{v}(0.4 \mathrm{keV})^{\mathrm{a}, \mathrm{b}}$ & $v L_{v}(1 \mathrm{keV})^{\mathrm{a}, \mathrm{b}}$ & $\alpha_{E S}^{b, c}$ & $v L_{v}(2 \mathrm{keV})^{\mathrm{a}, \mathrm{d}}$ & $v L_{v}(1325 \AA)^{\mathrm{a}, \mathrm{b}}$ \\
\hline $\mathrm{NAB} 0205+024^{\mathrm{e}} \ldots \ldots$ & $8.07_{-2.09}^{+1.79}$ & $1.60_{-0.63}^{+0.33}$ & $2.8 \pm 0.5$ & $1.07_{-0.12}^{+0.07}$ & \\
\hline$f \ldots \ldots \ldots \ldots \ldots \ldots \ldots \ldots$ & $5.53_{-0.60}^{+1.49}$ & $0.78_{-0.30}^{+0.30}$ & & $1.18_{-0.09}^{+0.09}$ & 2.2 \\
\hline PG $1211+143^{\mathrm{e}} \ldots \ldots \ldots$ & $2.46_{-0.65}^{+0.90}$ & $0.74_{-0.41}^{+0.33}$ & $2.3_{-0.3}^{+1.1}$ & $0.56_{-0.06}^{+0.10}$ & \\
\hline$f \ldots \ldots \ldots+\cdots \cdots \cdots \cdots$ & $1.64_{-0.25}^{+0.74}$ & $0.25_{-0.10}^{+0.19}$ & & $0.62_{-0.07}^{+0.08}$ & 0.6 \\
\hline Mrk $205^{e} \ldots \ldots \ldots \ldots$ & $1.15_{-0.20}^{+0.62}$ & $0.31_{-0.10}^{+0.35}$ & $2.3_{-0.7}^{+1.1}$ & $0.92_{-0.04}^{+0.05}$ & \\
\hline$f \ldots \ldots \ldots \ldots \ldots \ldots \ldots \ldots$ & $0.77_{-0.28}^{+0.34}$ & $0.124_{-0.065}^{+0.16}$ & & $0.94_{-0.06}^{+0.06}$ & 0.3 \\
\hline TON $1542^{\mathrm{e}} \ldots \ldots \ldots \ldots$ & $0.71_{-0.25}^{+0.43}$ & $0.135_{-0.076}^{+0.225}$ & $2.4_{-0.7}^{+1.6}$ & $0.44_{-0.02}^{+0.02}$ & \\
\hline$f \ldots \ldots \ldots \ldots \ldots \ldots \ldots \ldots$, & $0.41_{-0.07}^{+0.20}$ & $0.005_{-0.003}^{+0.009}$ & & $0.45_{-0.07}^{+0.07}$ & 0.8 \\
\hline PG $1244+026^{\mathrm{e}} \ldots \ldots \ldots$ & $0.72_{-0.09}^{+0.12}$ & $0.22_{-0.062}^{+0.013}$ & $2.3_{-0.6}^{+0.4}$ & $0.088_{-0.004}^{+0.012}$ & \\
\hline$f \ldots \ldots \ldots \ldots \ldots \ldots \ldots \ldots$, & $0.49_{-0.04}^{+0.16}$ & $0.090_{-0.032}^{+0.050}$ & & $0.099_{-0.015}^{+0.019}$ & 0.2 \\
\hline PG $1426+015^{\mathrm{e}} \ldots \ldots \ldots$ & $3.21_{-0.58}^{+0.49}$ & $1.69_{-0.66}^{+0.29}$ & $1.8_{-0.3}^{+0.4}$ & $1.43_{-0.09}^{+0.12}$ & \\
\hline$f \ldots \ldots \ldots \ldots \ldots \ldots \ldots \ldots$ & $2.222_{-0.25}^{+0.54}$ & $0.144_{-0.034}^{+0.115}$ & & $1.56_{-0.14}^{+0.14}$ & 0.7 \\
\hline
\end{tabular}

a Units of $10^{44} \mathrm{ergs} \mathrm{s}^{-1}$.

b Soft X-ray component.

${ }^{c} F_{\mathrm{y}} \propto v^{-\alpha_{\mathrm{ES}}}$.

d Total X-ray luminosity.

e Two power-law fit.

f Power-law + thermal bremsstrahlung fit. 
tent with each other, within the rather large errors. However, the EXOSAT best fit spectral indices are in all cases steeper by $\Delta \alpha \sim 1$ than the PSPC best fit indices, and the break energy of $\sim 0.6 \mathrm{keV}$ estimated by Comastri et al. (1992) falls short than the typical break energy of $1 \mathrm{keV}$ found in Paper I. There could therefore be a systematic offset between the EXOSAT and the PSPC measurements. We note that the Comastri et al. indices were obtained by fitting the data from two distinct experiments (the ME and the LE). Since the ME starts to be sensitive only above $1 \mathrm{keV}$, while the peak of the LE sensitivity is below this energy, the best-fit parameters could be largely affected by inaccuracies in the relative calibration of the two instruments. For example a soft component slope steeper than the actual one would be easily obtained if, given the same incident spectrum, the normalization in the LE were systematically higher than that in the ME (also see the discussions on the relative calibration of two instruments in Masnou et al. 1992, and in Paper I). On the other hand, the energy band and resolution of the PSPC allow a quite accurate determination of the shape of the soft component of bright quasars. In Paper I we showed that calibration uncertainties currently limit this ability and forced us to adopt very conservative errors on the parameters describing the soft $X$-ray component. We remark here that the offset between the EXOSAT and PSPC results is in all cases smaller than the statistical + systematic uncertainties.

Masnou et al. (1992) fitted the IPC/MPC data with a broken power-law model (with $N_{\mathrm{H}}$ fixed to the Galactic value) for a sample of seven quasars, two of which are in common with the present sample. For these two quasars they found a low-energy slope of $\alpha_{\mathrm{ES}}=2.6(\mathrm{NAB} 0205+024)$ and $\alpha_{\mathrm{ES}}=1.47$ (Mrk 205). These slopes are consistent with the ones obtained from our analysis (see Table 1).

Saxton et al. (1993) fitted the EXOSAT ME/LE data of a sample of 12 quasars parameterized the soft excess as a thermal bremsstrahlung model with a fixed temperature of $0.2 \mathrm{keV}$, close to the best fit temperature obtained fitting this model to the composite spectrum of the six quasars in our sample in Paper I.

\subsection{IR-Optical-UV Data}

Table 2 gives the infrared ( $3 \mu \mathrm{m})$, optical (5500 $\AA$ ), and UV (2500 and $1325 \AA$ ) monochromatic luminosities $\left(v L_{v}\right)$. When more than one infrared, optical or UV observation is available we report the maximum and minimum observed luminosities including errors, the number of observations and their date span. As a reference we also give in Table 2 the average $\alpha_{\text {ox }}$ of these quasars. The mean $\alpha_{\text {ox }}$ in the sample is 1.47 , slightly lower than the mean value of 1.525 found by Laor et al. (1994) for a complete sample of optically selected, low-redshift, radioquiet quasars (a difference of 0.06 in $\alpha_{\text {ox }}$ corresponds to a $40 \%$ difference in the optical to X-ray luminosity ratio). When available, the infrared, optical, and UV luminosities were extracted from the "Atlas of Quasar Energy Distributions" (Elvis et al. 1994a). Additional optical spectrophotometric data were included for NAB $0205+024$ and PG $1244+026$ (see below). The data from TON 1542 include optical data from Neugebauer et al. (1987), IR data from Rudy, Levan, \& RodriguezEspinosa (1982), and archival IUE spectra.

The $I U E$ data were corrected for the reduction in the efficiency of the cameras with the time (Bohlin \& Grillmar 1988). Following the procedures in Elvis et al. (1994a), the data were corrected for reddening, shifted to the rest frame and corrected for the contribution of the host galaxy using the galaxy template given in Table 16 of Elvis et al. (1994a). These authors showed that the contribution of the host galaxy can be impor$\operatorname{tant}(\gtrsim 20 \%)$ for $M_{V}<-25\left[v L_{v}(5500 \AA)<2 \times 10^{45}\right]$. Direct measurements of the host galaxy were used in two cases (NAB $0205+024$ and PG 1211+143). In two cases (Mrk 205 and PG $1426+015$ ) we used the $H$-band luminosity of the host galaxy in the Elvis et al. sample $\left[\log L(\mathrm{H})=44.6_{-0.6}^{+0.3}\right.$, but note that in Mrk 205 we modified the upper error bar, so as not to overpredict the total $H$-band luminosity]. In the remaining two cases the observed $H$-band luminosity is lower than the mean host galaxy luminosity in Elvis et al. (1994a), and we assumed therefore that the host galaxy luminosity in these objects is a little lower than the observed $H$ luminosity. In all cases we used highly conservative error bars on the host galaxy luminosity. Table 2 lists the host galaxy normalization in the $H$ band for each quasar. Mrk 205 was quoted as a "weak bump quasar" by McDowell et al. (1989), showing a very steep optical to UV slope, but this slope turns out to be consistent with the quasar average slope $\left(f_{v} \sim-0.1\right)$ if the contribution from the host galaxy is close to the mean value in Elvis et al. (1994a). The errors on the host galaxy luminosity have been

TABLE 2

INFRARED-ULTRAVIOLET OBSERVATIONS

\begin{tabular}{|c|c|c|c|c|c|c|c|}
\hline Name & Redshift & $\log v L(H)^{\mathrm{a}}$ of Galaxy & $\begin{array}{c}v L_{v}(3 \mu \mathrm{m}) \\
\text { (No.; Dates) }\end{array}$ & $\begin{array}{r}v L_{v}(5500 \AA) \\
\text { (No.; Dates) }\end{array}$ & $\begin{array}{c}v L_{v}(2500 \AA) \\
\text { (No.; Dates) }\end{array}$ & $\begin{array}{c}v L_{v}(1325 \AA) \\
\text { (No.; Dates) }\end{array}$ & $\alpha_{\text {ox }}{ }^{b}$ \\
\hline NAB $0205+024 \ldots \ldots$ & 0.155 & $44.19 \pm 0.04$ & $\begin{array}{l}9.4_{-0.9}^{+1.0} \\
1 ; 85 / 9\end{array}$ & $\begin{array}{l}8.1-11.5 \\
3 ; 78 / 2-91 / 9\end{array}$ & $\begin{array}{l}33 \pm 1 \\
1 ; 82 / 12\end{array}$ & $\begin{array}{l}31 \pm 4 \\
1 ; 82 / 12\end{array}$ & 1.57 \\
\hline PG $1211+143 \ldots \ldots \ldots$ & 0.085 & $43.36_{-0.23}^{+0.04}$ & $\begin{array}{l}10.9-14.7 \\
5 ; 81 / 5-88 / 4\end{array}$ & $\begin{array}{l}9.7-16.6 \\
6 ; 80 / 6-88 / 9\end{array}$ & $\begin{array}{l}23.4-27.8 \\
2 ; 85 / 12-87 / 2\end{array}$ & $\begin{array}{l}19.1-31.3 \\
2 ; 83 / 12-85 / 2\end{array}$ & 1.64 \\
\hline Mrk $205 \ldots \ldots \ldots \ldots$ & 0.070 & $44.60_{-0.6}^{+0.1 c}$ & $\begin{array}{l}1.7-4.2 \\
3 ; 76 / 6-85 / 6\end{array}$ & $\begin{array}{l}1.6_{-0.6}^{+1.5} \\
1 ; 72 / 5\end{array}$ & $\begin{array}{l}1.8-7.3 \\
2 ; 78 / 7-83 / 2\end{array}$ & $\begin{array}{l}3.3-6.1 \\
2 ; 78 / 7-83 / 2\end{array}$ & 1.27 \\
\hline TON $1542 \ldots \ldots \ldots \ldots$ & 0.064 & $44.48_{-0.6}^{+0.1 \mathrm{~d}}$ & $\begin{array}{l}3.17_{-0.38}^{+0.63} \\
1 ; 81 / 5\end{array}$ & $\begin{array}{l}2.61_{-0.31}^{+0.75} \\
1 ; 80 / 11\end{array}$ & $\begin{array}{l}7.5 \pm 0.2 \\
1 ; 82 / 5\end{array}$ & $\begin{array}{l}10.2 \pm 0.3 \\
1 ; 82 / 5\end{array}$ & 1.47 \\
\hline PG $1244+026 \ldots \ldots \ldots$ & 0.048 & $43.76_{-0.6}^{+0.1 \mathrm{~d}}$ & $\begin{array}{l}0.39_{-0.04}^{+0.07} \\
1 ; 83 / 2\end{array}$ & $\begin{array}{l}0.53-0.92 \\
2 ; 7 / 80-5 / 91\end{array}$ & $\begin{array}{l}1.06 \pm 0.05 \\
1 ; 83 / 2\end{array}$ & $\begin{array}{l}1.06 \pm 0.05 \\
1 ; 83 / 2\end{array}$ & 1.41 \\
\hline PG $1426+015 \ldots \ldots \ldots$ & 0.086 & $44.60_{-0.6}^{+0.3 c}$ & $\begin{array}{l}5.9-12.1 \\
5 ; 81 / 7-88 / 5\end{array}$ & $\begin{array}{l}2.6-7.3 \\
6 ; 80 / 7-88 / 9\end{array}$ & $\begin{array}{l}22.3-28.4 \\
2 ; 83 / 2-85 / 5\end{array}$ & $\begin{array}{l}24.9-42.8 \\
2 ; 83 / 2-85 / 5\end{array}$ & 1.48 \\
\hline
\end{tabular}

a Units of $10^{44} \mathrm{ergs} \mathrm{s}^{-1}$.

${ }^{\mathrm{b}} \alpha_{\mathrm{ox}}=\frac{\log [L(2500 \AA) / L(2 \mathrm{keV})]}{2.6057}$.

c Galaxy subtraction for TON 1542 used the mean values given in Elvis et al. $1994: L_{\mathrm{H}}=4_{-3}^{+4} \times 10^{44} \mathrm{ergs} \mathrm{s}^{-1} ; r_{e}=10_{-6}^{+3} \mathrm{kpc}$.

d Galaxy normalization chosen to match the rest frame $H$ luminosity. 
propagated to find the uncertainties on the luminosity at optical and UV frequencies

Optical spectrophotometry was obtained for two of the quasars: NAB 0205+024 and PG 1244+026; on the Multiple Mirror Telescope (MMT) using the MMT spectrograph and the red channel on 1991 September 15 and 1991 May 17, respectively (the PSPC observations of these quasars were performed in 1992 January and 1991 December, respectively). On both dates conditions were photometric. Two spectra were obtained, the first through a wide slit $\left(5^{\prime \prime} \times 180^{\prime \prime}\right)$ to ensure photometric accuracy and the second through a narrow slit $\left(1.5^{\prime \prime} \times 180^{\prime \prime}\right)$ for improved spectral-resolution $(\sim 20 \AA)$ and signal-to-noise. The 150 lines $\mathrm{mm}^{-1}$ grating was used yielding a wavelength range of $\sim 3800-7600 \AA$. The spectra were reduced in the standard manner using IRAF. The wide-slit observation was flux calibrated with reference to a nearby standard star observed sequentially with the quasar. The continuum shape and level of the narrow-slit spectrum were then normalized to agree with this flux calibrated spectrum. Continuum fluxes at $5500 \AA$ measured from these spectra are presented in Table 2. The spectra will be presented in a later paper.

The errors contain the evaluated uncertainty on all the above corrections, in addition to the statistical uncertainty. In the case of the IUE data a further $3 \%$ systematic uncertainty is folded into the errors (Bohlin 1980). The data in different bands are not simultaneous. Variations of a factor of $\sim 2$ were recorded for these quasars in optical and UV and of a factor of 5 in $\mathrm{X}$-ray. The uncertainty due to source variability is generally larger than the quoted (statistical + systematic) errors.

\section{MODELING THE INFRARED TO SOFT X-RAY SPECTRAL}

\section{ENERGY DISTRIBUTIONS OF QUASARS}

\subsection{Optically Thin Plasma Models 4.1.1. The Model}

Thermal bremsstrahlung at temperatures of $10^{5}-10^{7} \mathrm{~K}$ emitted by an optically thin gas cloud is a candidate mechanism to produce the OUV/soft X-ray bump in quasar spectra (Barvainis 1993). We calculate the free-free contribution for a spherical and isothermal cloud assuming that the cloud has radius $(R)$ and density $\left(n_{e}\right)$ consistent with an optical depth less than $1\left(\tau_{\text {eff }}=\left[\tau_{v}\left(\tau_{v}+\tau_{\text {es }}\right)\right]^{1 / 2}<1\right.$; where $\tau_{v}$ are the free-free and $\tau_{\text {es }}$ the electron scattering optical depths). The bremsstrahlung luminosity is calculated using (Rybicki \& Lightman 1979):

$$
\begin{aligned}
L_{v}=9.5 \times 10^{-38}\left(\frac{4}{3} \pi R^{3}\right) & n_{e}^{2} T^{-1 / 2} \\
& \times \exp \left(-\frac{h v}{k T}\right) g_{\mathrm{ff}} \operatorname{ergs~s}^{-1} \mathrm{~Hz}^{-1}
\end{aligned}
$$

where $g_{\mathrm{ff}}$ is the Gaunt factor calculated using an approximation given by Gronenschild \& Mewe (1978).

In the soft X-ray band the free-free emission from the ionized plasma represents only part of the total emission, since recombination, 2-photon, and line emission from the same plasma can also be important, depending on the temperature (Raymond \& Smith 1977). We calculated the 2-photon and recombination continua as in Raymond \& Smith (1977). We do not include line emission in our modeling but we discuss in the next sections how it can effect the spectral shape and intensity.

\subsubsection{Comparison with the Data}

We plot in Figure 2 the $3 \mu \mathrm{m}-2 \mathrm{keV}$ SEDs of the six quasars, galaxy subtracted and including only the soft X-ray com-

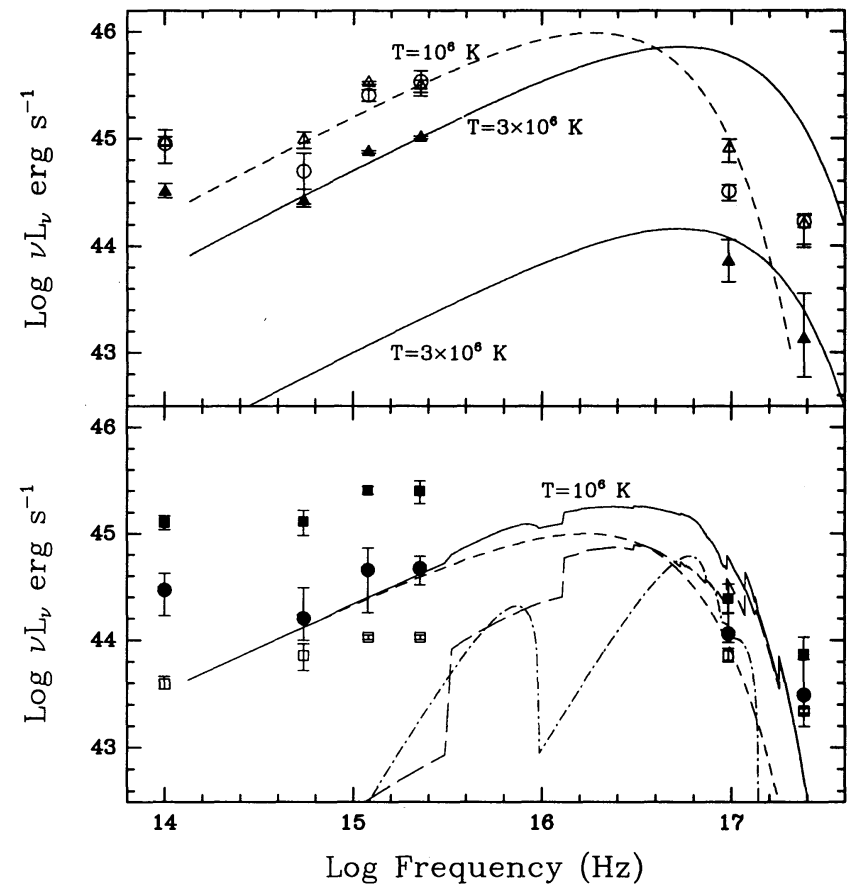

Fig. 2.-IR to soft X-ray SEDs of the six quasars (after the subtraction of the galaxy contribution and of the high-energy X-ray power law): NAB 0205+024: open triangles; PG 1211+143: filled squares; Mrk 205: filled circles; TON 1542: filled triangles; PG 1244+026: open squares; PG 1426+015: open circles. Upper panel: the solid lines represent the free-free continuum for $T=3 \times 10^{6} \mathrm{~K}$ and two normalizations; the dashed line represents the free-free continuum for $T=10^{6} \mathrm{~K}$. Lower panel: the solid line represents the sum of the continua from an optically thin plasma with $T=10^{6}$ $\mathbf{K}$; the short-dashed line is the free-free continuum; the long-dashed line is the recombination continuum; the dot-dashed line is the two-photon continuum.

ponent. On the upper panel we also plot free-free models, for $T=10^{6}$ and $T=3 \times 10^{6}$, that roughly encompass the observed SED. On the lower panel we plot the free-free, 2-photon and recombination continua, as well as the sum of these continua, for $T=10^{6} \mathrm{~K}$. At this temperature free-free emission dominates the OUV part of the spectrum but the harder recombination continuum dominates the soft X-ray part of the spectrum. Note the numerous, rather deep edges present in the total continuum.

Rather than attempt a formal fit of these models to the data, we make use of color diagrams to evaluate their flexibility and to evaluate the range of the parameters for which they are able to reproduce the data (also see Siemiginowska et al. 1995).

In Figure 3 we plot the soft X-ray color $\left[v L_{v}(1 \mathrm{keV}) / v L_{v}(0.4\right.$ $\mathrm{keV})$, soft component only], against the OUV color $\left[v L_{v}(1325\right.$ $\left.\AA) / v L_{v}(5500 \AA)\right]$ for the six quasars. The soft X-ray color is calculated using the $\mathrm{X}$-ray monochromatic luminosities in Table 2. The dotted line with open squares shows the predictions of pure bremsstrahlung models with temperatures in the range $10^{6}-10^{7} \mathrm{~K}$. The additional contribution of recombination and 2-photon continua would increase by a factor of 6 the continuum at $0.4 \mathrm{keV}$ and by a factor of 30 the continuum at $1 \mathrm{keV}$ for $T=10^{6} \mathrm{~K}$ thus increasing the soft X-ray color by a factor of 5 . For $T=10^{7} \mathrm{~K}$ the contribution of recombination and 2-photon continua would increase the flux at $0.4 \mathrm{keV}$ by a factor of 1.2 and the flux at $1 \mathrm{keV}$ by a factor of 1.4 , thus increasing the X-ray color by $9 \%$. Filled squares show soft $\mathrm{X}$-ray color predicted in these two cases.

The bremsstrahlung model can reproduce the observed soft $\mathrm{X}$-ray color for temperatures in the range $2-5 \times 10^{6} \mathrm{~K}(0.15-$ 


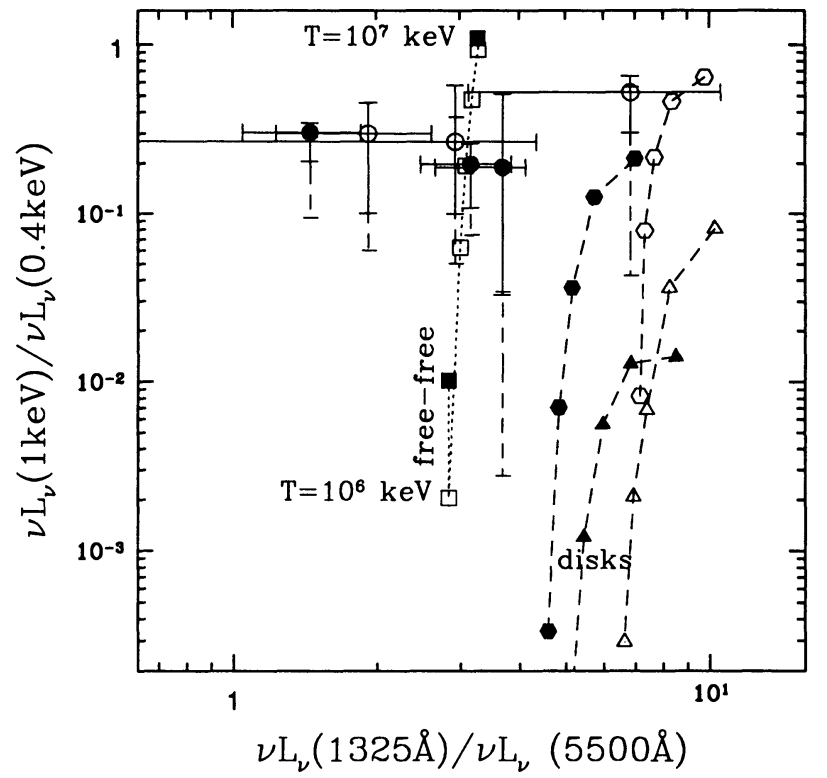

FiG. 3.-Soft X-ray color $\left[v L_{v}(1 \mathrm{keV}) / v L_{y}(0.4 \mathrm{keV})\right.$, soft component only], plotted against the OUV color $\left[v L_{v}(1325 \AA) / v L_{v}(5500 \AA)\right]$. Filled and open circles with error bars identify the six quasars, the dashed error bars indicate the error resulting from a power law plus free-free fit (Paper I). In these cases (open circles) (PG 1211+143, Mrk 205, and PG 1426+015) the quasars have been observed by IUE more than once and we plot the OUV color obtained using both the maximum and minimum optical and UV flux. The dotted line identifies the prediction of a pure free-free model as a function of temperature with open boxes indicating: 1, 2, 3, 5, $10 \times 10^{6} \mathrm{~K}$ (soft X-ray color increases with temperature). The filled boxes indicate the soft X-ray color predicted including the contribution of 2-photon and recombination continua for $T=10^{6}$ and $T=10^{7} \mathrm{~K}$. Dashed lines identify accretion disk models in a Kerr geometry. Filled hexagons and triangles indicate five disk inclinations, $\mu=$ $\cos \theta=1,0.75,0.5,0.2,0.1$ (soft X-ray color increases with inclination) with $M=10^{8} M_{\odot}$ and accretion rates $0.3 L_{\mathrm{Edd}}$ and $0.8 L_{\mathrm{Edd}}$. Open hexagons and triangles identify disk models with $M=10^{7} M_{\odot}$ for the same inclinations and accretion rates.

$0.4 \mathrm{keV}$ ). Including the contribution of 2-photon and recombination continua yields a lower limit of $\sim 1.5 \times 10^{6} \mathrm{~K}$ on the temperature. These models can also reproduce the observed OUV color.

In Table 1 we also list the upper limits on the $1325 \AA$ predicted luminosity calculated by extrapolating the best X-ray fit (for the power-law plus thermal bremsstrahlung model) into the UV. Because of the large extrapolation and the uncertainty on the contribution of the recombination and 2-photon continua in the X-ray band (which would lower the best fit temperature and raise the normalization, and hence produce higher UV luminosity), we assumed as upper limit on the predicted UV luminosity twice the best fit value. In Figure 4 we plot the observed $1325 \AA$ I luminosities versus the predicted ones. When more than one IUE observation is available we used the smallest observed $1325 \AA$ luminosity. Figure 4 shows that the model underpredicts the $1325 \AA$ luminosity by a factor 3 to 10 .

\subsubsection{Discussion}

Optically thin plasma models can reproduce the observed soft X-ray color for temperatures in the range $1.5-5 \times 10^{6} \mathrm{~K}$ $(0.13-0.4 \mathrm{keV})$. At these temperatures line emission can also be important in the X-ray band. The main emission lines, assuming collisional equilibrium, for temperatures $10^{6}<T<$ $5 \times 10^{6} \mathrm{~K}$, are the iron $L$ complex at about $0.9 \mathrm{keV}$ and the $\mathrm{O}$ VII and $\mathrm{O}$ VIII $\mathrm{K} \alpha$ lines at 0.57 and $0.65 \mathrm{keV}$. However, the ionization state of the soft X-ray emitting gas in quasar nuclei

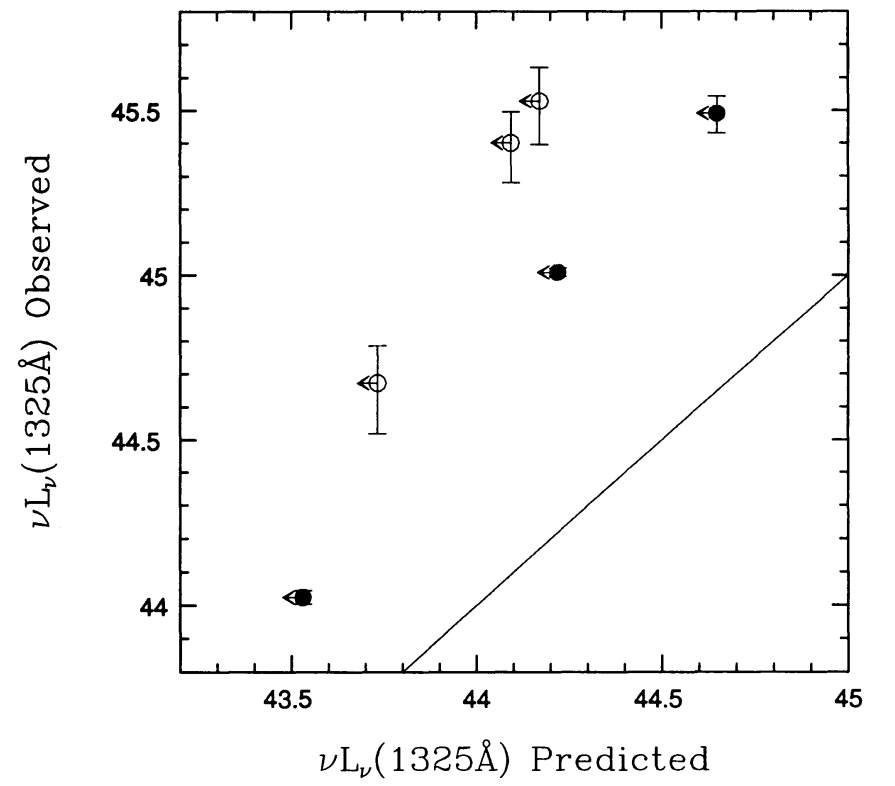

FIG. 4.-Observed $1325 \AA$ luminosity plotted against that predicted by the best-fit power-law plus thermal bremsstrahlung model for the X-ray spectrum of the six quasars. The predicted luminosity upper limits are twice the best-fit luminosity from $\mathrm{X}$-ray spectral fitting.

is unlikely to be controlled by collisions only, and photoionization is likely to be important, especially in the innermost regions close to the hard X-ray source. This strong radiation field could reduce the line emission intensities. Recent calculations by Kriss (1994) show that in the case of plasma in photoionization equilibrium the $\mathrm{O}$ vII and $\mathrm{O}$ vIII $\mathrm{K} \alpha$ lines are still very strong. Paper I limits the amount of line emission in the six quasars in the $0.5-1 \mathrm{keV}$ energy range (in most cases there is a deficit of counts with respect to a power-law model rather than an excess, see Figs. 1 and 3 in Paper I). It should be said however that emission features have been seen in the Einstein SSS spectra of some other quasars (Turner et al. 1992). In particular features, interpreted as oxygen lines in the framework of an ionized absorber model, have been recently seen in the X-ray spectrum of the Seyfert galaxy NGC 3783 (George, Turner, \& Netzer 1995). The lack of strong emission in the quasars discussed in this paper argues against emission from isothermal optically thin ionized gas as the main contributor to the soft X-ray spectrum of these sources (see Paper I, $\$ 3.5$ and 3.6), unless the soft X-ray emission lines are optically thick while the continuum is optically thin. Since at temperatures of interest $\left(T<10^{7} \mathrm{~K}\right)$ most of the cooling is in the emission lines, this requires high plasma densities. In some free-free models the assumed gas density is very high $\left(\sim 10^{15} \mathrm{~cm}^{-3}\right.$, case 2 in Barvainis 1993). Whether such a density is high enough to suppress line emission efficiently should be investigated in detail.

Optically thin plasma models predict OUV colors consistent with the observed ones (taking into account the large uncertainties due to source variability and the fact that the observations were not simultaneous). This color, however, is not very sensitive to temperature for $T \gtrsim 5 \times 10^{5} \mathrm{~K}$, i.e., in the range for which the models can explain the soft X-ray color $(\sim 15 \%$ change in the OUV color). Therefore, these models would not be able to produce the soft X-ray color higher than $\sim 0.001$ and at the same time give a spread in the OUV color. Detecting, through simultaneous observations in optical and UV, a 
large spread in the OUV color in a sample of quasars with strong soft excesses and soft X-ray color higher than $\sim 0.001$ would therefore be a critical test for the single-temperature free-free models.

The best-fit power-law plus free-free models to the PSPC data underpredicts the UV luminosity of the six quasars. The reason is that the fit forces a high temperature, locating the maximum of the emission in the soft X-ray, while the highest luminosities are actually observed in the UV band. Fitting the $\mathrm{X}$-ray data with the power-law plus free-free model while requiring that the predicted $1325 \AA$ luminosity matches the observed value, yields $\chi^{2}$ much worse than in the previous cases (by $\Delta \chi^{2}=20$ to 100 ) and temperatures between 0.05 and $0.13 \mathrm{keV}$ (the best fit temperature in the fits with free thermal component normalization is between 0.15 and $0.3 \mathrm{keV}$ in all cases). As mentioned before, for $T<10^{7} \mathrm{~K}$ the contribution of the recombination continuum would significantly harden the spectrum, but still not enough to explain the soft X-ray color for the temperatures which would fit the OUV part of the spectrum.

In summary, the single temperature optically thin plasma models are probably too simple to explain both the OUV and soft X-ray component of these quasars simultaneously. The strong constraints posed by the present data could be relaxed by dropping the hypothesis that the OUV and soft X-ray emission are dominated by a single component. Optically thin plasma at increasing temperatures could dominate the optical to soft X-ray quasar SED. This conclusion is somewhat ironic, since one of the most celebrated results of optically thin plasma models for the quasar blue bump (see, e.g., Barvainis 1993) is the natural explanation of the simultaneous variations at different wavelength, observed in a few, typically low-luminosity $\left(L_{\text {bol }}<10^{45}\right.$ ergs s $\left.^{-1}\right)$ quasars, due to a single physical component dominating the OUV emission.

Different frequencies dominated by components at different temperature is the distinguishing feature of another large class of models: the accretion disk models, which we discuss in turn.

\subsection{Accretion Disk Models}

\subsubsection{The Models}

We consider emission from a geometrically thin accretion disk (outer radius of 1000 Schwarzschild radii) around a supermassive $\left(10^{6}-10^{8} M_{\odot}\right)$ black hole with accretion rates $(\dot{m})$ corresponding to $0.01-0.08 L_{\text {Edd }}$ (the Eddington luminosity), assuming that the locally generated flux is emitted in the vertical direction only. Laor \& Netzer(1989) pointed out that this assumption could be unsafe for $\dot{m}>0.3$. In fact, for such high accretion rates disks become slim (Abramowicz et al. 1988), and additional cooling processes should be included (advective transport of energy in the radial direction. However, Szuszkiewicz et al. (1995) show that the spectra of thin and slim accretion disks differ significantly only when accretion rates are much higher than the critical one. We limited ourselves to the sub-Eddington regime only. The error introduced using our approach instead of the above more detailed calculations for $\dot{m}>0.3$ should be smaller than all other uncertainties present in the analysis.

The efficiency of converting the potential energy into radiation is assumed to be equal to 0.08 for a nonrotating (Schwarzschild) and to 0.324 (Laor \& Netzer 1989) for a maxi" mally rotating (Kerr) black hole. Equations for the disk structure are taken from Novikov \& Thorne (1973) and Page \& Thorne (1974). We calculated the spectrum using the method described by Czerny \& Elvis (1987, taking into account comments of Maraschi \& Molendi 1988; see also Czerny 1993) which includes electron scattering and Comptonization. For disk temperatures less than $10^{6} \mathrm{~K}$ free-bound transitions are important and the electron scattering and Compton effects are correspondingly less significant. It is difficult to estimate this contribution, since free-bound opacities depend on the ionization state of matter and on a large number of transitions of many different ions. Ross et al. (1992) calculated spectra emitted by a radiation-dominated disk (in the Schwarzschild geometry) including free-bound processes from hydrogen and helium. However, at high frequencies, free-bound opacities from metals may be more important and may influence the final spectra strongly and should be taken into account. This has not been accomplished yet. Since we are interested in the general shape of the soft X-ray spectra we use the free-bound approximation of Maraschi \& Molendi (1988). For rotating black holes we use the general relativistic transfer function of Laor, Netzer, \& Piran (1990).

Pure accretion disk models in a Schwarzschild geometry systematically underpredict the soft X-ray emission for any choice of parameters that reproduces the OUV observed luminosity so we will no longer consider these models.

We consider also two modifications of the pure accretion disk model, namely inclusion of an underlying power law, and inclusion of a hot corona above the disk (Czerny \& Elvis 1987).

\subsubsection{An IR-to-X-Ray Underlying Power Law}

It is well known that accretion disk models are falling too steeply to long wavelengths in the optical region. Many previous modeling attempts (Malkan \& Sargent 1982; Sun \& Malkan 1989; Laor 1990) have invoked an additional component in the form of an "underlying power law" that props up the disk flux in the optical and extends into the IR beneath the lumps clearly due to thermal dust emission (Clavel, Wamsteker, \& Glass 1989; Barvainis 1992). The normalization of this power law would be such that it extrapolates quite well into the X-ray region (Carleton et al. 1987; Elvis et al. 1986).

Independent evidence for such a power law is weak and even contradictory (see McAlary \& Rieke 1988; Green, Anderson, \& Ward 1992) and in at least one quasar (NGC 4051, Done et al. 1990) there is a strong evidence against a power-law component representing the majority of the emission in IR and X-rays (even if the IR-to-X-ray SED of NGC 4051 actually resembles a power law, see Fig. 6 in Done et al. 1990). Nevertheless, since we need additional flux in both the optical and the soft X-ray bands, the minimal assumption is to add the same component to each, and the simplest form to assume is a power law. This adds two additional parameters (slope and normalization of the power-law), but because the model cannot overpredict either the $3 \mu \mathrm{m}$ or hard X-ray luminosities it is also more constrained than a simple disk model, and so has predictive power.

\subsubsection{Comptonization in a Corona above the Disk}

A hot corona above a relatively cool accretion disk has been discussed by many authors (e.g., Liang \& Price 1977; Begelman, McKee, \& Shields 1983; Begelman \& McKee 1983; White \& Lightman 1989). The vertical thickness of the corona is small compared to its horizontal dimension. The corona is optically thin $\left(\tau_{\text {es }}<0.8\right)$ and has a high temperature $\left(T_{\text {cor }}\right.$, $10-50 \mathrm{keV}$ ) compared to that of the photons emitted by the disk. These photons are upscattered by the hot electrons, and the primary disk emission is modified in that a power law is produced at frequencies $v_{0} \ll v<k T_{\text {cor }} / h$ (with an exponential 
tail $\sim v^{3} \exp \left(-h v / k T_{\text {cor }}\right)$ for $v>k T_{\text {cor }} / h$; Sunyaev \& Titarchuk 1985; Górecki \& Wilczewski 1984; Zdziarski 1985). In the above expression $v_{0}$ is the typical frequency of the soft photons. We follow the simplified description of the process given by Sikora \& Zbyszewska (1986) (see also Czerny \& Zbyszewska 1991) in the Schwarzschild geometry to find the modified OUV-to-soft-X-ray disk spectrum.

The energy index of the power law of the Comptonized spectrum depends on the optical depth, temperature and geometry of the corona (Sunyaev \& Titarchuk 1985). In Figure 5 we plot the energy index of the power law of the Comptonized spectrum as a function of the corona temperature for three values of $\tau_{\mathrm{es}}$. The temperature dependence is rapid: for $k T_{\text {cor }}=50 \mathrm{keV}$ $\tau_{\text {es }}=0.25$ and 0.5 the power-law energy index is 2.0 and 1.5, respectively. When $k T_{\text {cor }}=10 \mathrm{keV}$ and $\tau_{\mathrm{es}}=0.5$ the power-law energy index is about 5. Corona models with low $\tau_{\text {es }}$ and $k T_{\text {cor }}<50 \mathrm{keV}$ cannot explain the hard (2-10 keV) spectrum of quasars, but only that below $\approx 2 \mathrm{keV}$. The hard X-ray spectrum requires high $\tau_{\text {es }}$ and high temperatures.

\subsubsection{Comparison with the Data}

Disk models in a Kerr geometry for two choices of black hole masses $\left(10^{7} M_{\odot}\right.$ and $\left.10^{8} M_{\odot}\right)$, accretion rates (Fig. $6 a$; $\dot{m}=0.1,0.8$ ) and inclination angles (Fig. $6 b ; \mu=\cos \theta=0.25$, 1) are compared with the six quasar SED in Figure 6. The models roughly encompass the observed SED.

In Figure 3 we plot the predicted soft X-ray and OUV colors for four families of the Kerr disk models spanning a typical range of parameters. Pure Kerr disk models cannot reproduce OUV color for the range of parameters for which they can match the observed soft X-ray color (with the exception of PG $1426+015)$ : they are too "blue." Only high inclination $(\mu=0.25)$, high accretion rate $(\dot{m}=0.8)$ models can reproduce the observed soft X-ray color.

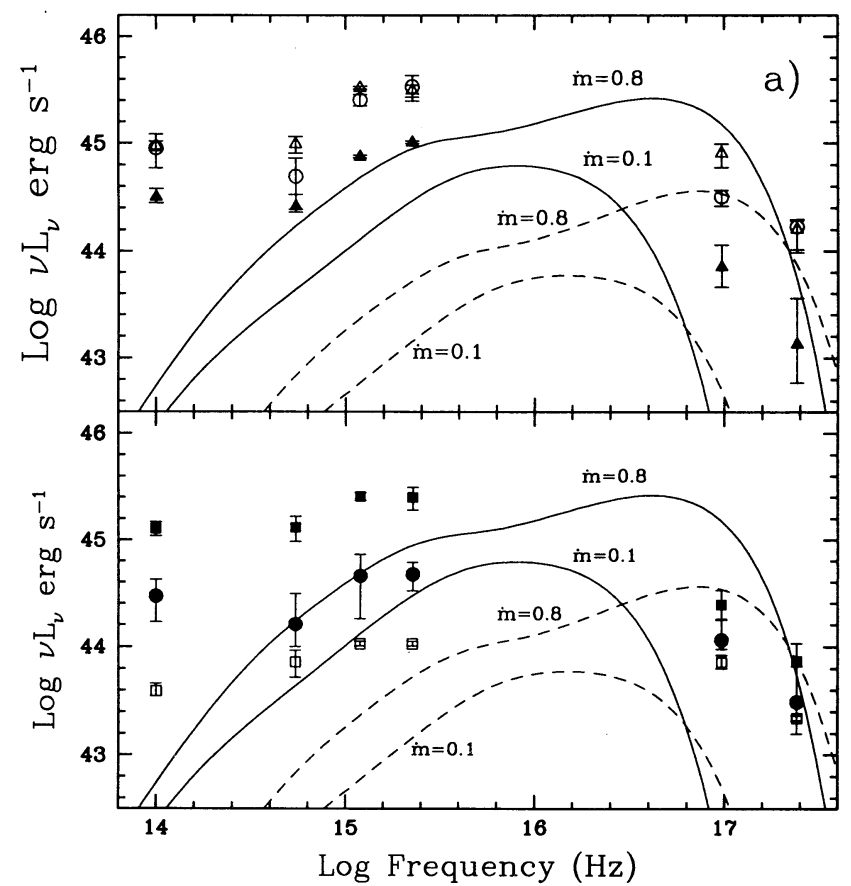

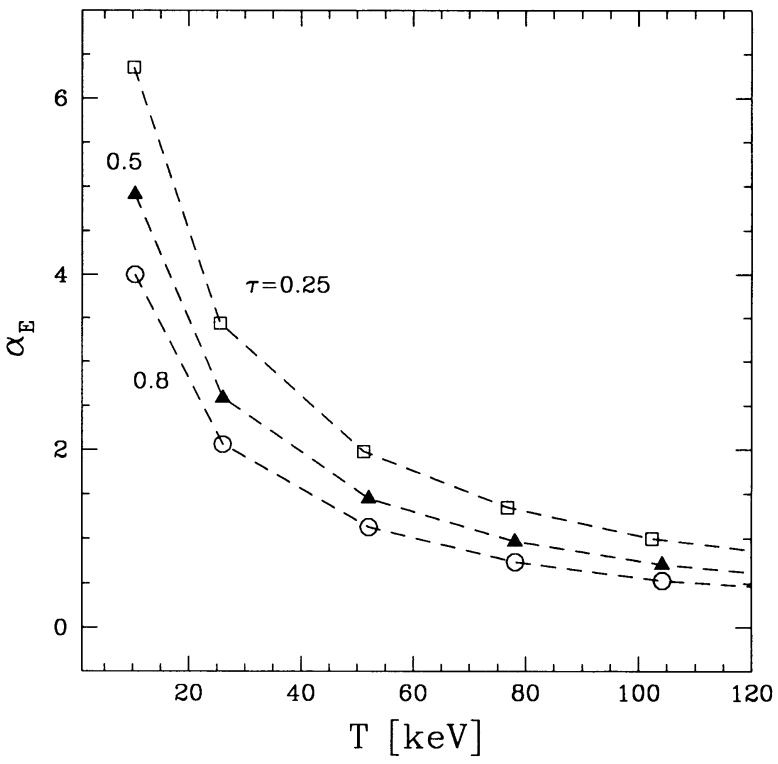

Fig. 5.-Spectral energy index of Comptonized spectrum in the corona model as a function of the temperature for three values of the optical depth.

In Figure 7 we plot the $0.4 \mathrm{keV}$ soft $\mathrm{X}$-ray component luminosity as a function of the $1325 \AA$ luminosity for pure Kerr disk models. The $M=10^{7} M_{\odot}$ models underpredict the 1325 A luminosity. The $M=10^{8} M_{\odot}$ models that could account for the soft X-ray color tend to overpredict the soft X-ray luminosity.

We study the effects of the inclusion of an underlying power law from IR to X-rays by using again the soft X-ray and the OUV colors. Figure 8 shows these colors with a set of models

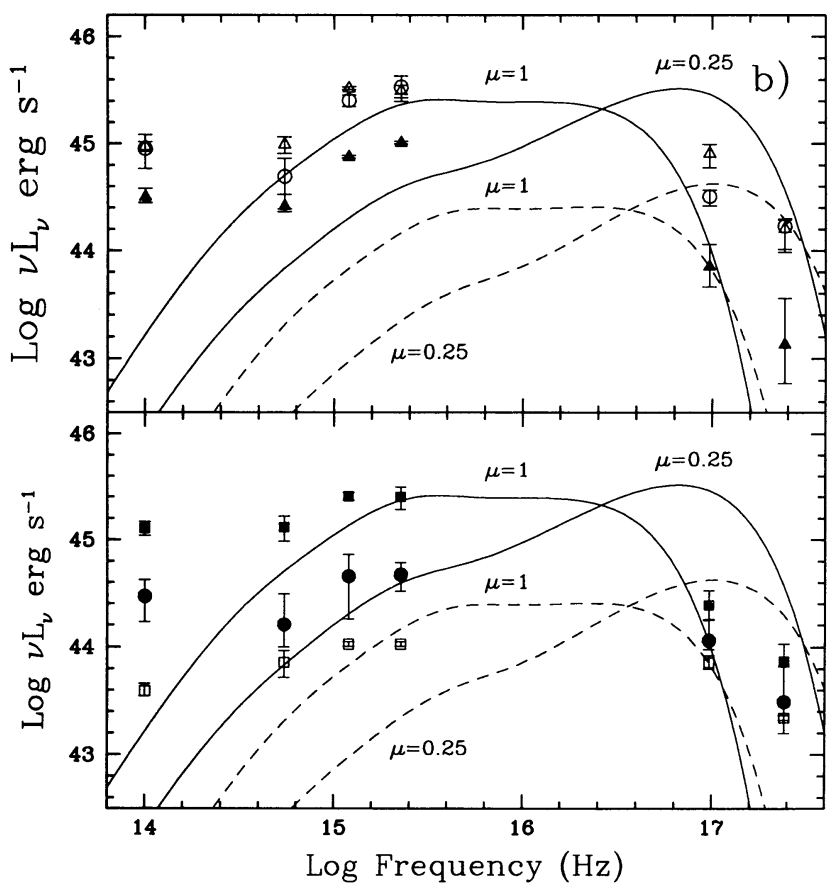

FIG. 6.-IR to soft X-ray SEDs of the six quasars (after the subtraction of the galaxy contribution and of the high-energy X-ray power law): NAB 0205+024: open triangles; PG 1211 + 143: filled squares; Mrk 205: filled circles; TON 1542: filled triangles; PG 1244 + 026: open squares; PG 1426+015: open circles. Solid lines in (a) represent accretion disk models in a Kerr geometry with $M=10^{8} M_{\odot}$, inclination $\mu=0.5$, and two accretion rates $(\dot{m}=0.1,0.8)$. Dashed lines identify Kerr disk models with $M=10^{7} M_{\odot}$ for the same accretion rates and inclinations. Solid lines in $(b)$ represents Kerr disk models with $M=10^{8} M_{\odot}$, accretion rate 0.8 the critical one and three disk inclinations, $\mu=1,0.5,0.2$. Dashed lines identify disk models with $M=10^{7} M_{\odot}$ for the same accretion rates and inclinations. 


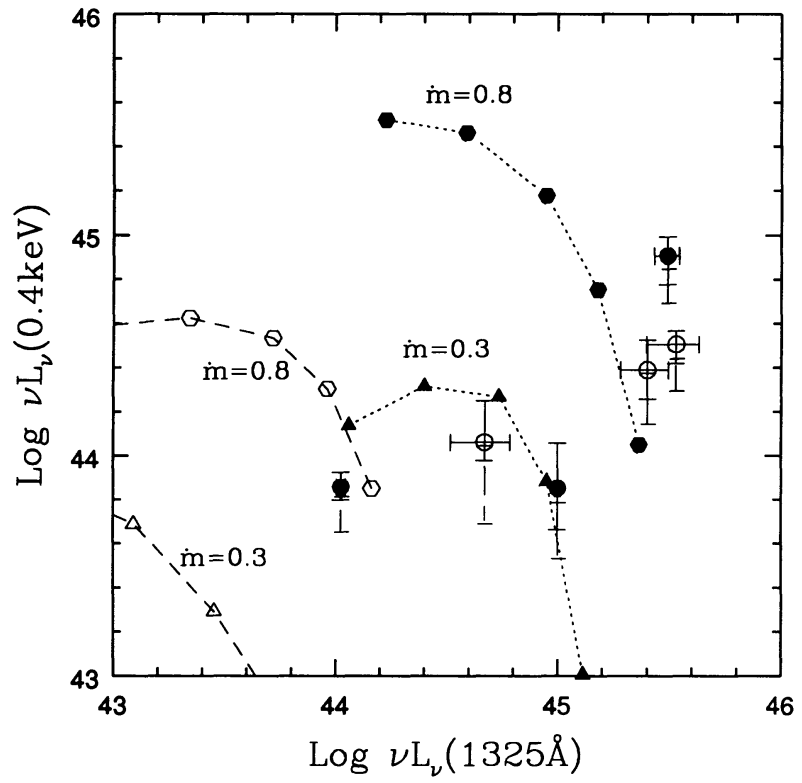

FIG. 7. $-0.4 \mathrm{keV}$ luminosity plotted against the $1325 \AA$ luminosity. Filled hexagons and triangles identify accretion disk models in a Kerr geometry with $M=10^{8} M_{\odot}, \dot{m}=0.3,0.8$, and five disk inclinations from $\mu=1$ to $\mu=0.1$. Open hexagons and triangles identify disk models with $M=10^{7} M_{\odot}$ for the same accretion rates and inclinations.

for two black hole masses $\left(10^{7} M_{\odot}\right.$ and $\left.10^{8} M_{\odot}\right), \dot{m}=0.3$ and $\mu=0.5, \alpha_{\mathrm{IRX}}=1.25$ and three normalizations of the power-law component. The model can reproduce both the soft X-ray and the OUV colors with typical disk parameters. The $3 \mu \mathrm{m}$ luminosity predicted by the above models is within the observed range (Fig. 9). Finally in Figure 10 we plot the $0.4 \mathrm{keV}$ soft $\mathrm{X}$-ray component luminosity as a function of the $1325 \AA$ luminosity. Also here in most cases the predicted luminosity match

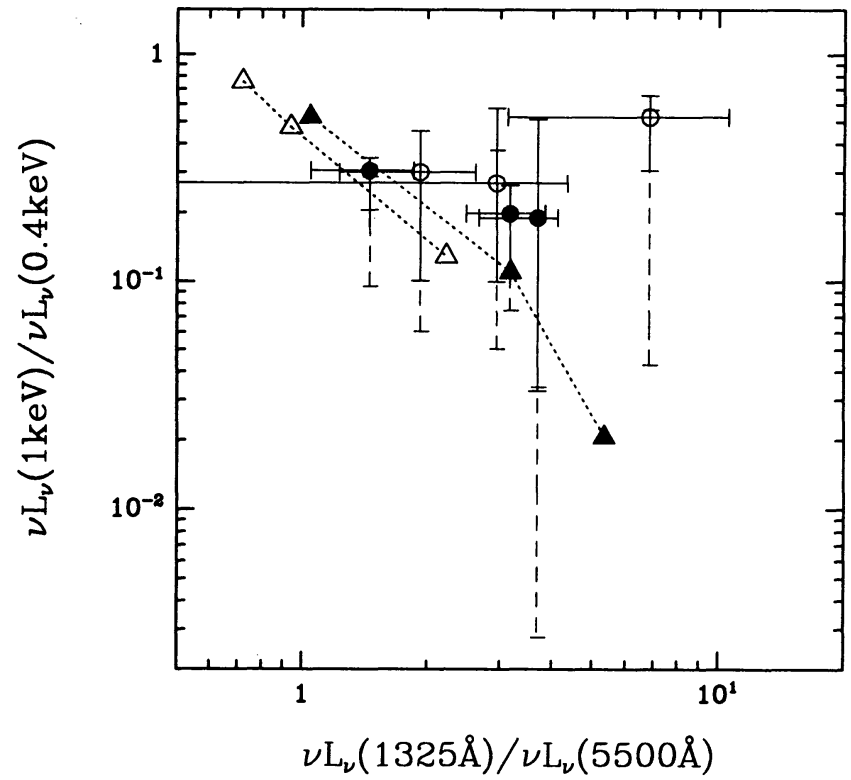

FIG. 8.-Soft X-ray color plotted against the OUV color as in Fig. 3. Filled triangles identify disk plus power-law models with $M=10^{8} M_{\odot}, \dot{m}=0.3$, disk inclination $\mu=0.5$, and three values of the power-law normalization. The power-law spectral index is fixed at 1.25 . Open triangles identify disk models with $M=10^{7} M_{\odot}$ for the same accretion rate, inclination, power-law spectral index and normalizations as in the previous case.

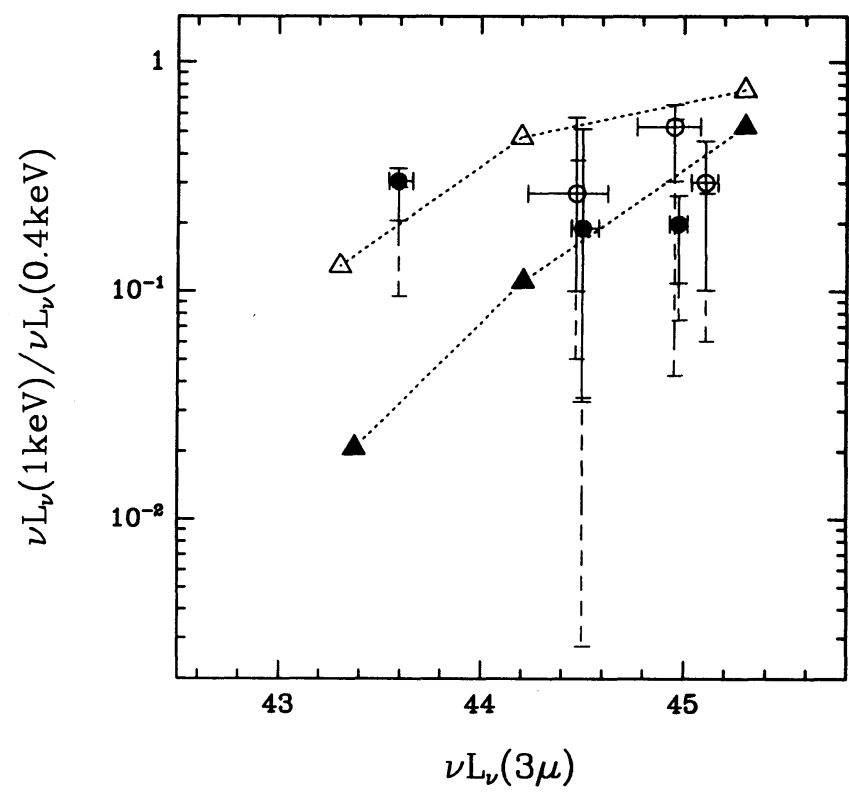

FIG. 9.- Soft X-ray color plotted against the $3 \mu \mathrm{m}$ luminosity. Filled triangles identify disk plus power-law models with $M=10^{8} M_{\odot}, \dot{m}=0.3$, disk inclination $\mu=0.5$, and three values of the power law normalization. The power-law energy index is fixed at 1.25 . Open triangles identify disk models with $M=10^{7} M_{\odot}$ for the same accretion rate, inclination, and power-law slope and normalizations as in the previous case.

that observed. We stress again that these models make use of a fixed accretion rate and a fixed inclination.

To study the effects of the inclusion of a hot corona we use the soft X-ray and OUV colors. These are plotted in Figure 11 along with the expectation of accretion disk plus corona models for two black hole masses, three accretion rates, 0.1 , 0.3 , and $0.8 \dot{m}, T_{\text {cor }}=10-50 \mathrm{keV}$ and $\tau_{\text {es }}=0.25-0.5$. These models are able to reproduce the observed soft X-ray and

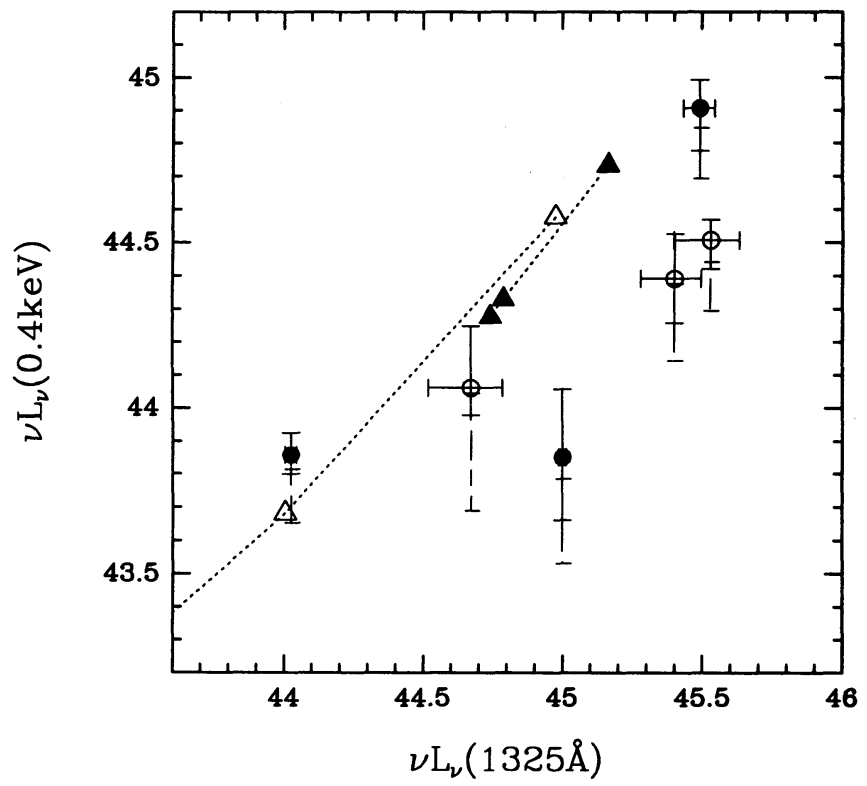

FIG. 10. $-0.4 \mathrm{keV}$ luminosity plotted against the $1325 \AA$ luminosity. Filled triangles identify disk plus power-law models with $M=10^{8} M_{\odot}, \dot{m}=0.3$, disk inclination $\mu=0.5$, and three values of the power-law normalization. The power-law slope is fixed at 1.25 . Open triangles identify disk models with $M=10^{7} M_{\odot}$ for the same accretion rate, inclination, and power law slope and normalizations as in the previous case. 


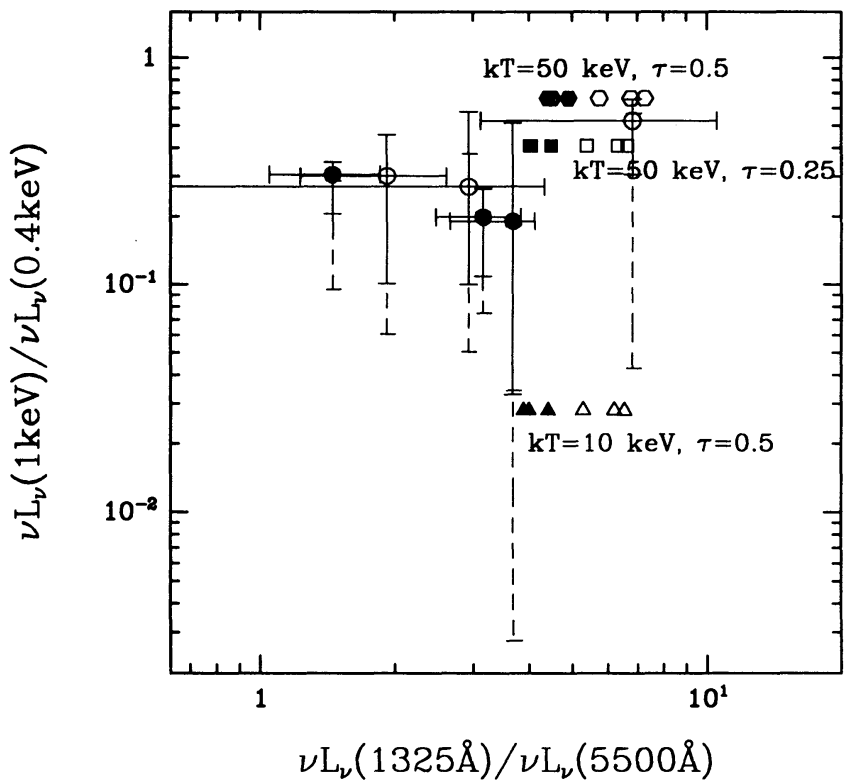

Fig. 11.-Soft X-ray color plotted against the OUV color as in Fig. 3. Filled hexagons identify accretion disk models in a Schwarzschild geometry with a hot corona with parameters: $M=10^{8} M_{\odot}, \dot{m}=0.1,0.3,0.8, k T=50$ $\mathrm{keV}$, and $\tau=0.5$. Filled squares identify models with $k T=50 \mathrm{keV}$ and $\tau=0.25$ and filled triangles models with $k T=10 \mathrm{keV}$ and $\tau=0.5$. Open symbols identify the same models but with $M=10^{7} M_{\odot}$. Higher accretion rates result in a higher UV to optical color.

OUV colors but they introduce two additional parameters with respect to the pure disk models.

\subsubsection{Discussion}

Accretion disk models have been widely used in the past to model the quasar blue bump. In spite of their popularity a clear proof that the majority of the OUV emission in the blue bump actually originates from a disk is still lacking. On the one hand there is both theoretical (e.g., Czerny 1993) and observational (e.g., double-peaked $\mathrm{H} \alpha$ lines in radio-galaxies, Eracleous \& Halpern 1994; the broad iron $\mathrm{K} \alpha$ lines reportedly seen by $A S C A$ in a number of Seyfert 1 galaxies, Mushotzky et al. 1995; especially the double-peaked iron $\mathrm{K} \alpha$ line in MCG -5-23-16 reported by Weaver 1995) support to the idea that disks do exist in the innermost regions of quasars. On the other hand the UV spectra of quasars do not show characteristic signatures predicted by simple accretion disk models (e.g., the review of Kinney 1994).

We find that pure accretion disk models are not able to reproduce the observed optical-to-soft-X-ray SED for the blue bump and soft X-ray component simultaneously. Even to fit just three points (the luminosity at $1325 \AA 0.4$ and $1 \mathrm{keV}$ ), some fine tuning seems unavoidable. Furthermore the range of parameters which give reasonable predictions is particularly narrow and extreme: high inclination, high accretion rate, and a black hole mass $\sim 10^{8} M_{\odot}$. The reason for this apparent lack of flexibility of Kerr disk models is that to account for the strength and shape of the soft X-ray component the luminosity of the spectrum must peak in the soft X-ray region rather than a decade lower $\sim 10^{16} \mathrm{~Hz}$ as the data suggest. On the other hand, pure accretion disk models capable of reproducing the observed quasar UV luminosity are steeper in the soft X-ray region than the observed spectra.

Adding an IR-to-X-ray power law to the disk models readily produces colors and luminosities in the observed range of
soft-X-ray and OUV simultaneously. Furthermore this combination does not violate the $3 \mu \mathrm{m}$ or hard X-ray constraints. This success certainly does not prove the existence of an underlying power law, but does raise the possibility of its existence again.

A more physically based additional component is a hot corona above the disk. The inclusion of this component, not surprisingly, help in hardening the spectra and in reproducing the soft X-ray color and luminosity.

Recently, Haardt \& Maraschi (1993) presented a model for the X-ray spectrum of quasars based on Comptonization of soft photons by a population of thermal electrons in a hot corona above a layer of cold reflecting matter. Their models reproduce well the $2-10 \mathrm{keV}$ spectrum of radio-quiet quasars, as well as the sharp steepening above $\sim 50-100 \mathrm{keV}$ found by SIGMA, OSSE, and ASCA in the spectra of a number of quasars (Jourdain et al. 1992; Maisack et al. 1993; Cameron et al. 1993; Elvis et al. 1994b). The soft X-ray spectrum, as inferred from Figures 4 and 5 in Haardt \& Maraschi (1993), breaks sharply around a characteristic energy that depends mainly on the temperature of the cold reflecting layer, which in turn depends on the physical parameters of the system (the mass of the central object, the size of the emitting region, and the luminosity in units of $L_{\mathrm{Edd}}$ ). For a black hole mass of $10^{6}$ $M_{\odot}, L=L_{\mathrm{Edd}}$, and a size for the X-ray emitting region of $7 \boldsymbol{R}_{\boldsymbol{S}}$, the temperature is $\sim 50 \mathrm{eV}$ and the break energy is around 1 $\mathrm{keV}$. The slopes below and above the break energy depend on the temperature of the corona, its optical depth and the inclination of the system to the line of sight. For low optical depth $(\tau=0.01)$ the $\Delta \alpha_{E}$ between the soft and hard slopes is very large for any inclination: it is large (5-6) for face on systems and reduces to zero in the case of $\tau=0.2$ and nearly edge-on systems. Our analysis (see Paper I) suggests a break energy at 1 $\mathrm{keV}$ and a $\Delta \alpha_{\mathrm{E}}=0.5-1$. To reproduce our observations the Haardt and Maraschi models would then require a small black hole mass and a peculiar inclination. It appears therefore that these models suffer from problems similar to those of pure disk models.

Another possible mechanism for hardening the soft X-ray spectrum from an accretion disk is via irradiation of its surface by an external X-ray source. Irradiation of the disk not only modifies the ionization state of the disk surface and produces an additional soft X-ray component (Ross \& Fabian 1993; Matt, Fabian, \& Ross 1993; Życki et al. 1994), but also affects the vertical structure of the disk. In addition, the outer parts of the disk will also be influenced by external irradiation (by the $\mathrm{X}$-rays and by the UV spectrum generated at small radii, if the fractional disk thickness increases outward and/or if relativistic deflection of the light is important, as in disks around a rotating black hole (Cunningham 1976). This may modify the OUV slope (Malkan 1991), and irradiation may be the cause for the flattening of the spectrum in the OUV band with respect to pure disk models and of the wide range of observed OUV slopes.

Ross \& Fabian calculated the reflection spectrum from a slab of gas at $R=7 R_{\mathrm{S}}$ under the assumption that the illuminating hard $\mathrm{X}$-ray flux is equal to the soft flux locally produced in the slab. They found that the ionization parameter of the gas determines the emerging X-ray spectrum. In their model the ionization parameter $\xi$ is directly linked to the accretion rate $\left(\xi \propto \dot{m}^{3}\right)$. Most recently Matt et al. (1993) generalized this approach calculating the UV and X-ray spectrum from an accretion disk illuminated by a central X-ray source. In the models of Ross \& Fabian the spectrum flattens by about 
$\Delta \alpha_{E} \approx 0.4$ from the $0.3-2 \mathrm{keV}$ region to the $2-20 \mathrm{keV}$ region, for $0.15<f<0.3$ and for a black hole mass of $10^{8}-10^{9} M_{\odot}$. A similar result is inferred from Figures 4 and 12 in Matt et al. (1993) for an illuminated accretion disk in a Schwarzschild geometry with a black hole mass of $10^{9} M_{\odot}$ and $\dot{m} \lesssim 0.4$.

The average PSPC spectral index of low optical luminosity $\left(M_{B}>-23\right)$, radio-quiet quasars without strong absorption ( 28 objects in the Walter \& Fink 1993 sample) is 1.44 , which implies a $\Delta \alpha_{E}$ with respect to the $2-10 \mathrm{keV}$ spectrum similar to that predicted by Ross and Fabian. On the other hand in the same sample the average spectral index of the radio-quiet quasars with $M_{B}<-23$ (17 objects) is 1.64 (similar to the average slope of the radio-quiet quasars in Laor et al. 1994), steeper than in lower optical luminosity sources. Furthermore, in $30 \%$ of these objects and in half of the sources of the sample presented in this paper $\alpha_{E}>2$ (e.g., NAB 0205+025, PG $1211+143$, PG $1244+026$, see Paper I). If the $2-10 \mathrm{keV}$ spectrum of these quasars has a slope close to the mean Ginga slope in Williams et al. (1992), then a $\Delta \alpha_{E} \sim 1$ is implied, significantly larger than predicted by reflection. The reason is that the integrated intensity of the reflected spectrum in the soft X-ray band cannot be much greater than the intensity of the ionizing X-ray continuum at the same energies (unless the X-ray source is obscured in part, or strongly beamed toward the disk). Reflection might account for the soft component of low optical luminosity quasar, but probably it does not dominate the soft component of the high optical luminosity quasars in the sample presented in this paper. The luminosity locally generated (not reprocessed) is likely to be a non-negligible part of the total soft X-ray luminosity in these sources. PSPC calibration uncertainties presently limit the strength of this result.

\section{CONCLUSIONS}

We have compared the observed OUV to soft X-ray SED, of six, radio-quiet, low-redshift quasars with the predictions of several competing models. Our main results are as follows:

1. Isothermal optically thin continuum emission from an ionized plasma cannot explain simultaneously the soft X-ray color and the luminosity in the optical and UV of the six quasars. A distribution of temperatures would help in relaxing the strong constraints posed by the present data. While this is not inconceivable, this assumption is not justified by the model itself but represents an "a posteriori" tuning.

2. On the other hand, accretion disk models naturally predict that components at different temperature dominate dif- ferent frequencies. However, we find that pure disk models, even in a Kerr geometry, cannot account for the observed OUV and soft $X$-ray colors and luminosities. The rather flat soft X-ray component slope found by the PSPC requires high inclinations and high accretion rates, which in turn overestimate the soft X-ray luminosity, when producing the correct UV luminosity.

3. The soft X-ray color can be explained if the soft X-ray emission is dominated by an additional component. The assumption of an underlying power law component extending from the infrared $(3 \mu \mathrm{m})$ to the X-ray, can explain both the OUV and, soft X-ray colors and luminosities, while it does not overpredict the observed $3 \mu \mathrm{m}$ luminosity. The presence of an optically thin hot $(T \lesssim 50 \mathrm{keV})$ corona around the accretion disk can explain the soft $\mathrm{X}$-ray color and luminosity.

4. A promising physical model is one involving reflection from an ionized accretion disk. This might explain the observed soft X-ray spectrum and luminosity of quasars with a soft X-ray component not much stronger than the primary component. However, it cannot readily explain the large soft excesses found in at least half of the quasars in this sample.

The present analysis, and the absence of strong line features in the PSPC spectra noted in Paper I, argue against singletemperature, solar metal abundance, optically thin emission as the only contributor to the OUV-to-soft-X-ray SED of the quasars in the sample presented in this paper. Pure disk models also fail to describe the quasar SED. While modifications of both optically thin plasma models and pure disk models might account for the observed SED, we do not find any strong evidence that the OUV bump and the soft X-ray emission are one and the same component, and that this component is thermal in origin. The observed SED could be produced by different components dominating different wavelengths, or by nonthermal emission mechanisms.

We thank Ari Laor for kindly providing us with the transfer function code for the Kerr geometry and for many useful discussions, Bożena Czerny, Magda Zbyszewska, and Giorgio Matt for discussions about Comptonization, disk emission and reflection. We also thank the referee, Richard Barvainis, for his comments that contributed to improve the paper. This work was supported by NASA grants NAGW-2201 (LTSARP), NAG5-1872, NAG5-1883 and NAG5-1536 (ROSAT), and NASA contracts NAS5-30934 (RSDC), NAS5-30751 (HEAO2) and NAS8-39073 (ASC).

\section{REFERENCES}

Abramowicz, M. A., Czerny, B., Lasota, J. P., \& Szuszkiewicz, E. 1988, ApJ, 332,646

Barvainis, R. 1992, in AIP Conf. Proc. 254, Testing the AGN Paradigm, ed. S. S. Holt, S. G. Neff, \& C. M. Urry (New York: AIP), 129 . 1993, ApJ, 412, 513

Begelman, M. C., \& McKee, C. F. 1983, ApJ, 271, 89

Begelman, M. C., McKee, C. F., \& Shields, G. A. 1983, ApJ, 271, 70

Bohlin, R. C. 1980, A\&A 85, 1

Bohlin, R. C., \& Grillmar, C. J. 1988, ApJS, 68, 5487

Cameron, R. A., et al. 1993, in AIP Conf. Proc. 280, Compton Gamma-Ray Observatory, ed. M. Friedlander, N. Gehrels, \& D. J. Macomb (New York: AIP), 478

Carleton, N. P., Elvis, M., Fabbiano, G., Willner, S. P., Lawrence, A., \& Ward, M. 1987, ApJ, 318, 595

Clavel, J., Wamsteker, W., \& Glass, I. S. 1989, ApJ, 337, 236

Comastri, A., Setti, G., Zamorani, G., Elvis, M., Giommi, P., Wilkes, B. J., \& McDowell, J. C. M. 1992, ApJ, 384, 62

Cunningham, C. T. 1976, ApJ, 208, 534

Czerny, B. 1993, in IAU Symp. 159, Multi-wavelength Continuum Emission of

AGN, ed. T. J. L. Courvoisier \& A. Blecha (Dordrecht: Kluwer), 261
Czerny, B., \& Elvis, M. 1987, ApJ, 321, 305

Czerny, B., Jaroszyński, M., \& Czerny, M. 1994, MNRAS, 268, 135

Czerny, B., \& Zbyszewska, M. 1991, MNRAS, 249, 634

Done, C., et al. 1990, MNRAS, 243, 713

Elvis, M., Giommi, P., Wilkes, B. J., \& McDowell, J. C. 1991, ApJ, 378, 537

Elvis, M., Green, R. F., Bechtold, J., Schmidt, M., Neugebauer, B. T., Soifer, B. T., Matthews, K., \& Fabbiano, G. 1986, ApJ, 310, 291

Elvis, M., Matsuoka, M., Siemiginowska, A., Fiore, F., Mihara, T., \& Brinkmann, W. 1994b, ApJ, 436, L55

Elvis, M., Wilkes, B. J., McDowell, J. C., Green, R. F., Bechtold, J., Willner, S. P., Polomski, E., \& Cutri, R. 1994a, ApJS, 95, 1

Edelson, R., et al. 1995, ApJ, 438, 120

Eracleous, M., \& Halpern, J. P. 1994, ApJS, 90, 1

Fiore, F., \& Elvis, M. 1995, in High Energy Radiation from Galactic and Extra-galactic Black Holes, in press

Fiore, F., Elvis, M., McDowell, J. C., Siemiginowska, A., \& Wilkes, B. J. 1994, ApJ, 431, 515 (Paper I)

George, I., Turner, T. J., \& Netzer, H. 1995, ApJ, 438, L37

Górecki, A., \& Wilczewski, W. 1984, Acta Astron., 34, 141

Green, P. J., Anderson, S. F., \& Ward, M. J. 1992, MNRAS, 254, 30 
Haardt, F., \& Maraschi, L. 1993, ApJ, 413, 507

Jourdain, E., et al. 1992, A\&A, 256, L38

Kinney, A. L. 1994, in ASP Conf. Ser. 54, The Physics of Active Galaxies, ed.

G. V. Bicknell, M. A. Dopita, \& P. J. Quinn (San Francisco: ASP), 61

Kriss, G. A. 1994, BAAS, 26, No. 2, 879

II Laor, A. 1990, MNRAS, 246, 396

II Laor, A., Fiore, F., Elvis, E., Wilkes, B. J., \& McDowell, J. C. 1994, ApJ, 435,

Laor, A., \& Netzer, H. 1989, MNRAS, 238, 897

Laor, A., Netzer, H., \& Piran, T. 1990, MNRAS, 242, 560

Liang, E. P. T., \& Price, R. H. 1977, ApJ, 218, 249

Maisack, M., et al. 1993, ApJ, 407, L61

Malkan, M. A. 1988, Adv. Space Res., 8, No. 2-3, (2)49

1991, in Structure and Emission Properties of Accretion Disks, ed.

C. Bertout et al. (Gif-sur-Yvette: Éditions Frontières), 165

Malkan, M. A., \& Sargent, W. L. W. 1982, ApJ, 254, 22

Maraschi, L., \& Molendi, S. 1988, ApJ, 353, 452

Masnou, J.-L., Wilkes, B. J., Elvis, M., McDowell, J. C., \& Arnaud, K. A. 1992, A\&A, 253, 35

Matt, G., Fabian, A. C., \& Ross, R. R. 1993, MNRAS, 264, 839

McAlary, C. W., \& Rieke, G. H. 1988, ApJ, 333, 1

McDowell, J. C., Elvis, M., Wilkes, B. J., Willner, S. P., Oey, M. S., Polomski,

E., Bechtold, J., \& Green, R. F. 1989, ApJ, 345, L13

Mushotzky, R., et al. 1995, MNRAS, 272, L9

Neugebauer, G., Green, R. F., Matthews, K., Schmidt, B. T., Soifer, B. T., \& Bennet, J. 1987, ApJS, 63, 615

Novikov, I. D., \& Thorne, K. S. 1973, in Black Holes, ed. C. De Witt \& B. De Witt (New York: Gordon \& Breach), 347

O'Brien, P. T., Gondhalekar, P. M., \& Wilson, R. 1988, MNRAS, 233, 845

Page, D. N., \& Thorne, K. S. 1974, ApJ, 191, 499

Pfefferman, E., et al. 1987 Proc. SPIE, 733, 519

Pounds, K. A. 1994, in New Horizon of X-Ray Astronomy, ed. F. Makino \& T. Ohashi (Tokyo: Universal Academic Press)
Pounds, K. A., et al. 1993, MNRAS, 260, 77

Puchnarewicz, E. M., Mason, K. O., Siemiginowska, A., \& Pounds, K. A. 1995, MNRAS, in press

Raymond, J. C., \& Smith, B. W. 1977, ApJS, 35, 419

Ross, R. R., \& Fabian, A. C. 1993, MNRAS, 261, 74

Ross, R. R., Fabian, A. C., \& Mineshige, S. 1992, MNRAS, 258, 189

Rudy, R. J., LeVan, P. D., \& Rodriguez-Espinosa, J. M. 1982, AJ, 87, 598

Rybicki, G. B., \& Lightman, A. P. 1979, Radiative Processes in Astrophysics (New York: Wiley)

Saxton, R. D., Turner, M. J. L., Williams, O. R., Stewart, G. C., Ohashi, T., \& Kii, T. 1993, MNRAS, 262, 63

Shields, G. A. 1978, Nat, 272, 706

Siemiginowska, A., Kuhn, O., Elvis, M., Fiore, F., McDowell, J. C., \& Wilkes, B. J. 1995, ApJ, submitted

Sikora, M., \& Zbyszewska, M. 1986, Acta Astron., 36, 255

Sun, W.-H., \& Malkan, M. A. 1989, ApJ, 346, 68

Sunyaev, R. A., \& Titarchuk, L. G. 1985, A\&A, 143, 374

Szuszkiewicz, E., et al. 1995, in preparation

Trümper, J. 1983, Adv. Space Res., 2, No. 4, 241

Turner, T. J., \& Pounds, K. A. 1988, MNRAS, 232, 463 1989, MNRAS, 240, 833

Turner, T. J., Weaver, K. A., Mushotzky, R. F., Holt, S. S., \& Madejsky, G. M 1991, ApJ, 381, 85

Walter, R., \& Fink, H. H. 1993, A\&A, 274, 105

Walter, R., Orr, A., Courvoisier, T. J.-L., Fink, H. H., Makino, F., Otani, C., \& Wamsteker, W. 1995, A\&A, in press

Weaver, K. 1994, BAAS, 26, No. 4, 1500

White, T. R., \& Lightman, A. P. 1989, ApJ, 340, 1024

Wilkes, B. J., \& Elvis, M. 1987, ApJ, 323, 243

Williams, O. R., et al. 1992, ApJ, 389, 157

Zdziarski, A. A. 1985, ApJ, 289, 514

Życki, P. T., Krolik, J. H., Zdziarski, A. A., \& Kallman, T. R. 1994, ApJ, 437, 597 